\title{
Spin Filtering and Entanglement Swapping through Coherent Evolution of a Single Quantum Dot
}

\author{
Jose Garcia Coello, ${ }^{1}$ Abolfazl Bayat, ${ }^{1}$ Sougato Bose, ${ }^{1}$ John H. Jefferson, ${ }^{2}$ and Charles E. Creffield ${ }^{3}$ \\ ${ }^{1}$ Department of Physics and Astronomy, University College London, Gower Street, London WC1E 6BT, United Kingdom \\ ${ }^{2}$ QinetiQ, Emerging Technologies Group, St Andrews Road, Malvern, Worcestershire WR14 3PS, United Kingdom \\ ${ }^{3}$ Departamento de Física de Materiales, Universidad Complutense de Madrid, E-28040, Madrid, Spain
}

(Received 3 March 2010; revised manuscript received 12 June 2010; published 17 August 2010)

\begin{abstract}
We exploit the nondissipative dynamics of a pair of electrons in a large square quantum dot to perform singlet-triplet spin measurement through a single charge detection and show how this may be used for entanglement swapping and teleportation. The method is also used to generate the Affleck-Kennedy-LiebTasaki ground state, a further resource for quantum computation. We justify, and derive analytic results for, an effective charge-spin Hamiltonian which is valid over a wide range of parameters and agrees well with exact numerical results of a realistic effective-mass model. Our analysis also indicates that the method is robust to the choice of dot-size and initialization errors, as well as decoherence.
\end{abstract}

DOI: 10.1103/PhysRevLett.105.080502

Introduction.-Realizing quantum information and computation tasks in solid state systems, particularly quantum dots (QDs), has attracted a lot of interest in recent years. Electron spins in QDs are promising candidates for the physical implementation of a qubit [1] due to their long coherence times [2]. Initialization, manipulation, and readout of electron spins have already been demonstrated $[3,4]$ and ideas exist for quantum gates based on single qubits encoded in two QDs [5]. As it is timely for "proof of principle" demonstrations of multiqubit processes, it would be highly desirable to establish a coherent two qubit process in a single quantum dot.

Bell measurement is a key ingredient that makes possible some important tasks such as teleportation [6] and entanglement swapping [7]. In this Letter, we propose a mechanism for singlet-triplet measurement based on the coherent dynamics of two electrons in a large square QD, followed by a single charge detection. Such spin filtering will give a perfect Bell measurement in the $S_{z}=0$ subspace of two spins. This projection is made possible due to the existence of a ground manifold of two singlets and two triplets, separated from higher-lying states by a large energy gap. To a very good approximation this enables the low-energy coherent dynamics to be confined to the ground manifold in which the singlets rotate around the quantum dot whereas the triplets are frozen at their initial locations. By initializing the system in an unentangled superposition state, we are then able to project onto a singlet or triplet state simply by a charge measurement to detect whether or not the charge has moved during the evolution. We use this property to propose some quantum information applications such as entanglement swapping and generating the Affleck-Kennedy-Lieb-Tasaki (AKLT) state, which is a resource for measurement-based quantum computation [8].

From a practical perspective a large square QD is easier to fabricate than a small one and will also be modeled more accurately by our effective Hamiltonian, since the energy gap between the ground manifold and the lowest excited
PACS numbers: 03.67.Lx, 03.65.Yz, 72.25.-b

states increases rapidly with dot size. On the other hand, as the absolute sizes of the singlet-triplet splitting in the ground manifold fall exponentially with dot size, large QDs have slower operation times and are more susceptible to errors. There is thus a trade-off between these factors, favoring QDs of intermediate size. Our simulations show that for square QDs of $L=200-800 \mathrm{~nm}$ our effective Hamiltonian is sufficiently accurate and operates at frequencies within the range achieved in Ref. [9].

Effective Hamiltonian. - We consider a system of two electrons held in a square semiconductor QD with a hardwall boundary, which can be realized in experiment by gating a two-dimensional electron gas at a heterojunction interface. The spectrum of this system is determined by the competition between the kinetic energy $\left(\sim 1 / L^{2}\right)$ of the electrons and the Coulomb repulsion $(\sim 1 / L)$ between them. In small QDs the kinetic term dominates, and the charge density peaks at the center of the dot (like noninteracting particles). Conversely in large dots, when the Coulomb interaction dominates, the energy of the system is minimized by the electrons localizing in space to minimize the electrostatic interaction energy.

The square QD is modeled by

$$
H=\frac{-\hbar^{2}}{2 m^{*}}\left[\nabla_{1}^{2}+\nabla_{2}^{2}\right]+V\left(\mathbf{r}_{1}\right)+V\left(\mathbf{r}_{2}\right)+\frac{e^{2}}{4 \pi \varepsilon\left|\mathbf{r}_{1}-\mathbf{r}_{2}\right|},
$$

where $V(\mathbf{r})$ is the confining potential and $m^{*}$ is the electron effective mass. We choose this to be hard wall with exact square symmetry, though our results will not qualitatively change under small deviations from perfect symmetry. The last term in Eq. (1) represents the Coulomb repulsion between the two electrons. In the strongly correlated regime, in which the size of the square is large compared with the effective Bohr radius $a_{B}(\sim 10 \mathrm{~nm}$ in GaAs), eigenstates of this simple Hamiltonian are extremely demanding to obtain exactly. We show in Fig. 1(a) the lowlying energy spectrum of a GaAs QD with $L=800 \mathrm{~nm}$, 
obtained by diagonalizing the Hamiltonian of Eq. (1). We see that two degenerate triplets $(|n\rangle, n=3,4, \ldots, 8)$ sit approximately midway between two singlets $\left(\left|S_{1(2)}\right\rangle\right)$, while all these 8 states are separated from the next multiplet of eigenstates by a relatively large gap. The charge distribution for the ground state $\left|S_{1}\right\rangle$ is shown in Fig. 1(b), and clearly shows how the charge density strongly peaks near the corners of the QD. One can better appreciate the form of the states by defining linear combinations of the two singlets

$$
\begin{aligned}
& |1\rangle=\left(\left|S_{1}\right\rangle+\left|S_{2}\right\rangle\right) / \sqrt{2}=\left|\Phi_{1}^{S}\right\rangle\left|\psi^{-}\right\rangle, \\
& |2\rangle=\left(\left|S_{1}\right\rangle-\left|S_{2}\right\rangle\right) / \sqrt{2}=\left|\Phi_{2}^{S}\right\rangle\left|\psi^{-}\right\rangle,
\end{aligned}
$$

where $\left|\psi^{-}\right\rangle=(|\uparrow \downarrow\rangle-|\downarrow \uparrow\rangle) / \sqrt{2}$ is the singlet spinor and $\left|\Phi_{1(2)}^{S}\right\rangle$ is the symmetric spatial component of the twoelectron wave function. In Figs. 1(c) and 1(d) we plot the charge distribution of these states, clearly showing how they are localized at diagonally opposite corners of the QD. For the triplets we adopt a similar labeling scheme

$$
\begin{array}{cc}
|3\rangle=\left|\Phi_{1}^{A}\right\rangle\left|\psi^{+}\right\rangle, \quad|4\rangle=\left|\Phi_{2}^{A}\right\rangle\left|\psi^{+}\right\rangle, \quad|5\rangle=\left|\Phi_{1}^{A}\right\rangle|\uparrow \uparrow\rangle, \\
|6\rangle=\left|\Phi_{2}^{A}\right\rangle|\uparrow \uparrow\rangle, \quad|7\rangle=\left|\Phi_{1}^{A}\right\rangle|\downarrow \downarrow\rangle, \quad|8\rangle=\left|\Phi_{2}^{A}\right\rangle|\downarrow \downarrow\rangle,
\end{array}
$$

where $\left|\psi^{+}\right\rangle=(|\uparrow \downarrow\rangle+|\downarrow \uparrow\rangle) / \sqrt{2}$ and $\left|\Phi_{1}^{A}\right\rangle\left(\left|\Phi_{2}^{A}\right\rangle\right)$ is the antisymmetric charge distribution, which resembles that of the states $|1\rangle(|2\rangle)$, being peaked at the same sites $b d$ $(a c)$. Note that while the triplets $|n\rangle(n=3,4, \ldots, 8)$ are eigenvectors of $H$, the singlets $|1\rangle$ and $|2\rangle$ are not.

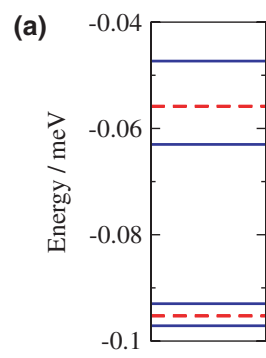

(c)

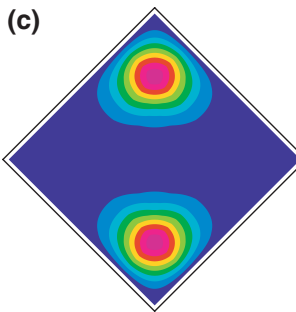

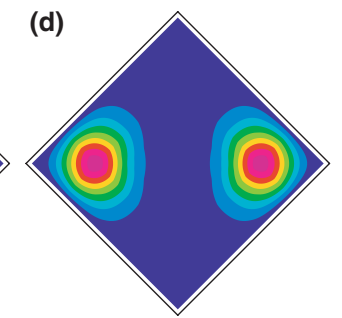

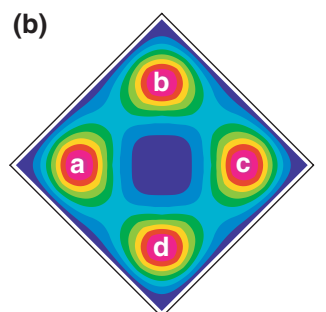

FIG. 1 (color online). Eigensystem of a GaAs dot with $L=$ $800 \mathrm{~nm}$. (a) The lowest two multiplets of states; singlets are shown with solid (blue) lines, triplets with dashed (red) lines. (b) Charge distribution of the ground state with peaks labeled abcd near the dot corners. (c) Charge distribution of the symmetrized singlet state $|1\rangle$, localized about $b d$. (d) Charge distribution of the antisymmetrized singlet state $|2\rangle$, localized about $a c$.
We can immediately write down an effective Hamiltonian for the low-lying energy eigenstates

$$
H_{\mathrm{eff}}=-\Delta_{1}\left|S_{1}\right\rangle\left\langle S_{1}\left|+\Delta_{2}\right| S_{2}\right\rangle\left\langle S_{2}\left|+E_{0} \sum_{n=1}^{8}\right| n\right\rangle\langle n|,
$$

where $E_{0}$ is the energy of the two degenerate triplets and $\Delta_{1}\left(\Delta_{2}\right)$ is the energy separation between the triplets and $\left|S_{1}\right\rangle\left(\left|S_{2}\right\rangle\right)$. By restricting ourselves to the ground manifold, and using the sum rule $\sum_{n=1}^{8}|n\rangle\langle n|=I$, the effective Hamiltonian may be written in the charge-spin form

$$
H_{\mathrm{eff}}=E_{0} I-\Delta(|1\rangle\langle 2|+| 2\rangle\langle 1|)+J\left(\mathbf{s}_{1} \cdot \mathbf{s}_{2}-1 / 4\right)
$$

where $J=\left(\Delta_{2}-\Delta_{1}\right) / 2$ and $\Delta=\left(\Delta_{1}+\Delta_{2}\right) / 2$. This has the simple physical interpretation that the Coulomb repulsion pushes the electrons to diagonally opposite corners, giving two charge states for each combination of spin.

Dynamics.-We now consider the time evolution of two electrons injected into the square dot so that one is located near $a$ and the other near $c$ [as labeled in Fig. 1(b)]. This could be achieved in principle using surface gates as shown schematically in Fig. 2. Initially an electron is localized in each of the small dots adjacent to the large dots. These electrons are then transferred to the large dot by lowering barriers using gates $G_{1}, G_{8}$ and $G_{4}, G_{5}$, which are subsequently restored to their previous potentials after electron transfer has completed. If both electrons have the same spin, i.e., total $S_{z}= \pm 1$, then this spin will not subsequently change with time under the coherent evolution of the Hamiltonian (7) and the two electrons will therefore remain close to their parent corners. However, if the two injected electrons are of opposite spin, then the state after injection will be an equal superposition of a singlet state and an $S_{z}=0$ triplet state, which will subsequently change with time. Let us consider the state in which a spin-up electron is injected at corner $a$ and a spin-down electron at corner $c$. We may approximate this state by

$$
|\psi(0)\rangle=\frac{|1\rangle+|3\rangle}{\sqrt{2}}=\frac{\left|\Phi_{1}^{S}\right\rangle+\left|\Phi_{1}^{A}\right\rangle}{\sqrt{2}}|\uparrow \downarrow\rangle-\frac{\left|\Phi_{1}^{S}\right\rangle-\left|\Phi_{1}^{A}\right\rangle}{\sqrt{2}}|\downarrow \uparrow\rangle .
$$

Note that both components correspond to spin-up at $a$ and spin-down at $c$ since $\Phi_{1}^{S}+\Phi_{1}^{A} \sim 0$ except when $\mathbf{r}_{1} \sim \mathbf{r}_{a}$, $\mathbf{r}_{2} \sim \mathbf{r}_{c}$ and $\Phi_{1}^{S}-\Phi_{1}^{A} \sim 0$ except when $\mathbf{r}_{1} \sim \mathbf{r}_{c}, \mathbf{r}_{2} \sim \mathbf{r}_{a}$. Hence this state is unentangled. Under the Hamiltonian (7), the time evolution of $|\psi(0)\rangle$ can be determined analytically as

$$
|\psi(t)\rangle=\frac{e^{-i E_{0} t}}{\sqrt{2}}\left[e^{i J t}(\cos (\Delta t)|1\rangle+i \sin (\Delta t)|2\rangle)+|3\rangle\right],
$$

choosing units with $\hbar=1$. We see directly that at time $t^{*}=\pi / 2 \Delta$, for which $\sin \left(t^{*} \Delta\right)=1$, we have an equal superposition of the states $|2\rangle$ and $|3\rangle$. At time $t^{*}$, therefore, a simple single charge detection at any corner (let us say $b$ ) will project $\left|\psi\left(t^{*}\right)\right\rangle$ into a singlet (with the electrons in 


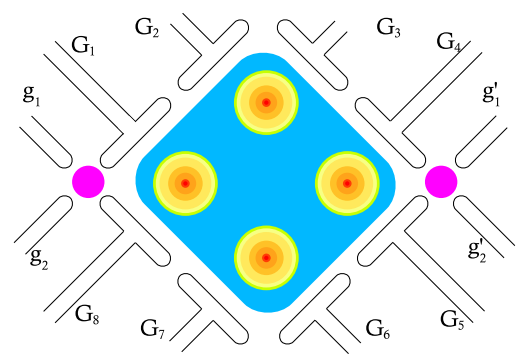

FIG. 2 (color online). Gate structure for a large QD (central shaded square), connected to two smaller QDs (pink circles).

corners $b$ and $d$ ) or a triplet (with electrons remaining in corners $a$ and $c$ ). Hence, if we project the state into a singlet then it oscillates between corners $b d$ and $a c$. Conversely, if we project it to the triplet then it is frozen in the corners $a c$.

The probability of detecting the singlet state at time $t$, starting in the $S_{z}=0$ subspace, is $P_{2}=|\langle 2 \mid \psi(t)\rangle|^{2}=$ $\frac{1}{2} \sin ^{2} \Delta t$. Thus $P_{2}$ oscillates harmonically with maximum probability $1 / 2$ but independently of the exchange, $J$. This independence of $J$ implies that our method of "filtering" the singlet by measurement is robust to the size of the dot, for which the ratio $J / \Delta$ falls exponentially with increasing dot size [10]. This is not the case for other overlaps. For example, the probability of finding the initial state is $|\langle\psi(0) \mid \psi(t)\rangle|^{2}=\left(1+\cos ^{2} \Delta t+2 \cos J t \cos \Delta t\right) / 4$, which shows that only for special cases (e.g., $J=0$ ) does the system return to its starting state.

Applications.-The ability to make singlet-triplet measurements enables entanglement swapping, or equivalently, teleportation. To achieve these we generate two singlet pairs outside a square QD as shown in Fig. 3(a). These pairs may be generated via surface gates in a similar fashion to those shown in Fig. 2 in which electrons are transferred from the surrounding 2D electron gas reservoir. The singlets are formed simply by cooling the system [4]. We then push one electron from each singlet pair into the big square QD as shown in Fig. 3(b). We now have two electrons in the corners $a$ and $c$ in the square $\mathrm{QD}$, and after time $t^{*}$ we measure the charge at one corner. With probability of $1 / 4$, the state of the electrons in the square QD collapses to a singlet at sites $b d$. In this case the two remaining electrons in the small QDs get entangled as another singlet, as shown in Fig. 3(c). This process is called entanglement swapping (or the teleportation of entanglement) and generates entanglement between distant particles. This scheme can be scaled up through a geometry shown in Fig. 3(d) where a series of empty square QDs are arranged between small QDs containing electron singlet pairs. By pushing one electron from each small QD to its neighboring square QD, one makes all small QDs empty except the two which terminate the array, that each hold one electron. Dynamical singlet-triplet measurement on all the square QDs generates a singlet between the electrons (a)

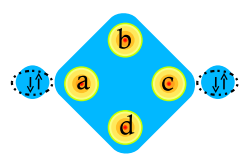

(c)

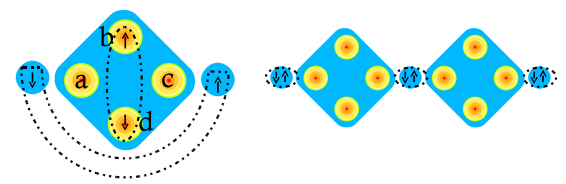

FIG. 3 (color online). (a) Two small QDs, with a singlet pair in each, beside a large square QD (dashed lines denote entanglement); (b) one electron from each singlet is pushed into the square QD; (c) entanglement swapping; (d) scaling up the system.

held in the terminating small dots when the result of all measurements is singlet. The probability of having this is $(1 / 4)^{N}$, where $N$ is the number of square QDs.

When the result of measurement in Fig. 3(b) is a triplet, rather than a singlet, we can generate the so-called AKLT state [11]. Originally this was introduced as the ground state of the AKLT Hamiltonian [11], which models the interaction of a series of spin- 1 particles with two spin- $1 / 2$ particles at the boundaries of a chain. The AKLT ground state can be generated by again starting with a series of spin-1/2 singlets in small QDs, but this time projecting two particles of neighboring singlets into a triplet to represent their spin- 1 nature. This occurs with probability $3 / 4$ when the result of the measurement in Fig. 3(b) is a triplet. This can also be scaled up with the geometry shown in Fig. 3(d), where the probability of success is $(3 / 4)^{N}$ that all square QD states will be in a triplet state. The AKLT state can be used as a resource for quantum computation [8].

Gate errors.-In any realistic situation these conditions will not be met, and in particular the starting state of Eq. (8) will contain small admixtures of the other states in the ground manifold and excited singlet states. These admixtures will increase with decreasing dot size but should still give small errors for $L>10 a_{B}$, for example. We can derive expressions for the fidelity starting with a more realistic state, $|\tilde{\psi}(0)\rangle$. This could be produced, for example, by applying a positive potential to gates located near the sites $a$ and $c$. In the numerical calculations, this was modeled by dividing the square dot into four quadrants and applying a constant positive potential to the two diagonally opposite quadrants that contain the corners $a$ and $c$. In this scheme setting the gating potential to $0.1 \mathrm{~V}$ yields values for the overlap $\langle\tilde{\psi}(0) \mid \psi(0)\rangle$ of $0.80,0.940$, and 0.97 for QDs of $L=200,800$, and $1200 \mathrm{~nm}$, respectively, which are reasonably close to unity, and could be enhanced further by using more elaborate gating potentials. We may derive an expression for the fidelity with this more realistic initial state by expanding $|\tilde{\psi}(0)\rangle$ in terms of $|\psi(0)\rangle,(|1\rangle-|3\rangle) \sqrt{2}$ 
TABLE I. Physical parameters for a GaAs QD.

\begin{tabular}{rccccc}
\hline \hline $\mathrm{L}(\mathrm{nm})$ & $\Delta(\mathrm{meV})$ & $J(\mathrm{meV})$ & $|\alpha|^{2}$ & $|\beta|^{2}$ & $E_{h f}(\mu \mathrm{eV})$ \\
\hline 100 & 0.814 & -0.243 & 0.441 & $5.23 \times 10^{-2}$ & 1.74 \\
200 & 0.145 & $-4.363 \times 10^{-2}$ & 0.445 & $3.63 \times 10^{-5}$ & $7.76 \times 10^{-1}$ \\
400 & $2.11 \times 10^{-2}$ & $-5.05 \times 10^{-3}$ & 0.420 & $1.21 \times 10^{-3}$ & $3.88 \times 10^{-1}$ \\
800 & $2.08 \times 10^{-3}$ & $-2.20 \times 10^{-4}$ & 0.453 & $2.78 \times 10^{-4}$ & $1.94 \times 10^{-1}$ \\
1600 & $9.34 \times 10^{-5}$ & $-1.66 \times 10^{-6}$ & 0.490 & $6.02 \times 10^{-6}$ & $9.69 \times 10^{-2}$ \\
\hline \hline
\end{tabular}

and the remaining eigenstates of the full effectivemass Hamiltonian. After time evolution and projection onto $|2\rangle$ we obtain $P_{2}^{e}=|\langle 2 \mid \tilde{\psi}(t)\rangle|^{2}=(\alpha \sin \Delta t)^{2}-$ $2 \alpha \beta \sin J t \sin \Delta t+\beta^{2}$, where $\alpha=\langle 1 \mid \tilde{\psi}(0)\rangle$ and $\beta=$ $\langle 2 \mid \tilde{\psi}(0)\rangle$. Note that $P_{2}^{e}$ is independent of excited states, and since $|\alpha|^{2} \sim 1 / 2,|\beta|^{2} \sim 0$, it is robust to gate errors. This is illustrated in Table I where we see only small deviations from the ideal $P_{2}$, even for the smallest dot of $L=100 \mathrm{~nm}$.

Charge measurement.-Typical values of $t^{*}$ being of the order of nanoseconds for our parameters (see Table I), are challenging to measure directly in experiment. For practical implementation, we propose a similar scheme to Ref. [9], which is able to achieve an acceptable time resolution. At the moment of measurement we restore the quadrant gate potentials (used previously to initialize the system) to freeze the dynamics of the electrons. A strong charge measurement at one of the corners of the QD can then be made to project the state into a singlet or triplet.

Charge dephasing.-Charge dephasing reduces the coherence between $|1\rangle$ and $|2\rangle$ in Eq. (9), but since our measurement projects onto these states anyway, it does not fundamentally affect our scheme. By damping the sinusoidal oscillations between $|1\rangle$ and $|2\rangle$, charge dephasing also reduces $P_{2}\left(t^{*}\right)=\left|\left\langle 2 \mid \psi\left(t^{*}\right)\right\rangle\right|^{2}$ such that in the extreme case of very strong decoherence it goes to $1 / 4$. In this case if $|2\rangle$ is detected successfully the scheme is completed as before, giving entanglement swapping. Otherwise, we end up with a superposition of $|1\rangle$ and $|3\rangle$, as in the initial state (except for the amplitude of $|1\rangle$ being reduced), which again undergoes damped oscillations. By repeating this process one can reliably (with exponential improvement according to number of trials) discriminate between singlets and triplets in the initial state. However, due to our fast dynamics this extreme case is very unlikely. As an example, for $L=400 \mathrm{~nm}$ we have $t^{*}=0.3 \mathrm{~ns}$, which is safely below the dephasing time $T_{2} \sim 1-2 \mathrm{~ns}$ in a system with comparable size [9].

Hyperfine interaction. - This can be estimated by replacing the effect of the nuclei with an effective magnetic field $\vec{B}$ coupled to the electron spin as $H_{h}=g \mu_{B} \vec{B} \cdot \vec{\sigma}$, where $\vec{\sigma}$ are the Pauli matrices. $\vec{B}$ has a Gaussian random distribu- tion with a variance $B_{n}$ [2]. Because of the fast evolution, the first maximum of $P_{2}(t)$ is relatively unaffected for typical energy values of $E_{h f}=g \mu_{B} B_{n}$, given in Table I. As an example, for $L=400 \mathrm{~nm}\left(t^{*}=0.3 \mathrm{~ns}\right)$, the decoherence time scale of hyperfine interaction is $10.7 \mathrm{~ns}$.

Conclusions. - We have shown that the dynamics of a pair of electrons in a large square QD can be used to perform singlet-triplet spin measurement using just a single charge detection. This is accessible to current technology and, unlike previous schemes, it is fast, deterministic, and coherent. Repeating the singlet-triplet measurement to a chain enables entanglement swap over a distance and the generation of the AKLT state in a way that would enable proof of principle quantum information experiments. Furthermore, coherent evolution of the system is considerably faster than the dephasing time $T_{2}$ imposed by the hyperfine interaction. Our low-energy analytic description is valid for a wide range of parameters, particularly for typical experimental values of the QD parameters.

A. B. and S. B. are supported by the EPSRC. J. G. C. was supported by the QIPIRC. S. B. acknowledges the Royal Society and the Wolfson Foundation. C.E.C. was supported by the MICINN (Spain) through Grant No. FIS2007-65723, and the Ramón y Cajal Program. J.H.J. acknowledges support from the UK Ministry of Defence.

[1] D. Loss and D. P. DiVincenzo, Phys. Rev. A 57, 120 (1998).

[2] J. M. Taylor et al., Phys. Rev. B 76, 035315 (2007).

[3] R. Hanson et al., Phys. Rev. Lett. 94, 196802 (2005).

[4] J. R. Petta, Science 309, 2180 (2005).

[5] R. Hanson and G. Burkard, Phys. Rev. Lett. 98, 050502 (2007).

[6] C. H. Bennett et al., Phys. Rev. Lett. 70, 1895 (1993).

[7] M. Zukowski et al., Phys. Rev. Lett. 71, 4287 (1993).

[8] G. K. Brennen and A. Miyake, Phys. Rev. Lett. 101, 010502 (2008).

[9] G. Shinkai et al., Phys. Rev. Lett. 103, 056802 (2009).

[10] C. E. Creffield et al., Phys. Rev. B 59, 10719 (1999).

[11] I. Affleck et al., Commun. Math. Phys. 115, 477 (1988). 